\title{
Properties of the P Cygni wind found using the Self Absorption Curve method
}

\author{
G. Muratorio ${ }^{1}$, N. Markova ${ }^{2}$, M. Friedjung ${ }^{3}$, and G. Israelian ${ }^{4}$ \\ ${ }^{1}$ Laboratoire d'Astrophysique de Marseille, 2 place le Verrier, 13248 Marseille Cedex 04, France \\ e-mail: muratorio@observatoire.cnrs-mrs.fr \\ 2 Institute of Astronomy and Isaac Newton Institute of Chile Bulgarian Branch, \\ National Astronomical Observatory, PO box 136, 4700 Smoljan, Bulgaria \\ e-mail: rozhen@mbox.digsys.bg \\ 3 Institut d'Astrophysique, 98 bis Boulevard Arago, 75014 Paris, France \\ e-mail: fried@iap.fr \\ 4 Instituto de Astrofisica de Canarias, Via Lactea, 38200 La Laguna, Tenerife, Spain \\ e-mail: gil@ll.iac.es
}

Received 29 March 2002 / Accepted 13 May 2002

\begin{abstract}
We have used the optical lines of N II and Fe III to study the wind of the luminous blue variable P Cyg. This was performed by applying a version of the Self Absorption Curve (SAC) method, involving few assumptions, to lines whose flux can be measured. A rather surprising result was obtained; the lines of more excited multiplets without blue shifted absorption components appear to be optically thick, while the lines of the most excited multiplets may show some indications of being optically thicker than the lines of less excited ones. Explanations of such effects are discussed, including possible inhomogeneities in the wind.
\end{abstract}

Key words. stars: early-type - stars: winds, outflows - stars: individual: P Cyg

\section{Introduction}

The observational history of the hypergiant P Cygni begun about 400 years ago, when it was discovered by Willem Blaeu as a nova-like object. Because of its spectral and photometric characteristics the star is considered to be the prototype of the P Cygni-type stars (PCT), as defined by Lamers (1986), and as one of the prototypes of the giant eruption Luminous Blue Variables (Humphreys 1999). In addition, recent studies (Markova et al. 2001; de Groot et al. 2001) showed that over the last 15 years P Cygni has behaved as a typical weak S Doradus variable in a short S Dor phase (van Genderen 2001). More information about this enigmatic object can be found elsewhere (Israelian \& de Groot 1999, ASP Conf Ser., 233).

Stahl et al. (1993) reported the presence of a number of pure emission lines in addition to the dominant $\mathrm{P}$ Cygni-type lines in the optical spectrum of P Cygni. Later Markova \& de Groot (1997) showed, comparing line-identification lists from various observational epochs, that the optical emission spectrum of $\mathrm{P}$ Cygni was much richer and intense in the mid nineties of the nineteenth century, than 60 years ago. In particular, the authors noted that more than $70 \%$ of the pure emission lines appear to be of recent origin. Among these are forbidden lines of Fe II, Ni II, Ti II, Fe III and N II; high excitation lines of Fe III and

Send offprint requests to: M. Friedjung, e-mail: fried@iap.fr
$\mathrm{N}$ II and lines of low and medium excitation of Si II. If not due to observational selection caused by the continuous improvement of the signal-to-noise ratio of photographic spectra during the last century, this result might indicate the presence of a very long-term variation in the wind+photospheric properties of the star. In this context, it seems worthwhile to try to obtain additional information on the nature and the origin of P Cygni's emission spectrum using methods not used up to now, such as for instance the SAC (Self-Absorption Curve) method developed by Friedjung \& Muratorio (1987). This method involves a semi-empirical analysis of emission line spectra of complex atoms and ions, without assuming detailed models for the objects emitting these spectra. By means of the SAC method valuable information (e.g. the self absorption effects, the level population laws and population anomalies) has been obtained for the Fe II emission spectrum of many stars of different types, such as AG Car (Muratorio \& Friedjung 1988), the VV Cep star KQ Pup (Muratorio et al. 1992), while procedures for using the method is described by Baratta et al. (1998). Use of the SAC method can require however a few hypotheses about the relative populations of levels inside the same spectroscopic term and the distribution of self-absorption relative to that of the emission of different lines in different parts of the line emitting region. The second of these hypotheses was in any case not needed in the present work. 
The main purpose of our study is to apply the SAC method to the Fe III and N II emission spectra of P Cygni. In this way we can hope to gain additional information about the nature and the origin of these spectra and thus to get a deeper insight into the physics of the star itself. The observational material is described in Sect. 2, where a number of problems concerning the derivation of the net emission equivalent widths of the studied lines are also discussed and resolved. Section 3 presents the results obtained through the SAC analysis while Sect. 4 deals with the interpretation of these results.

\section{Observations and data reduction. EW measurements}

The present analysis is based on one spectrum taken at the Heidelberg Observatory on August 91991 with a fibre echelle spectrograph attached to a telescope of the $70 \mathrm{~cm}$ class (Stahl et al. 1995). An EEV CCD with $1252 \times 770$ pixels of $22 \mu$ size was used as a detector. The spectrum covers an interval of $2700 \AA$, from $\lambda 4001$ to $\lambda 6773$, with a spectral resolution of 12000 and a signal to noise ratio of 333 (Stahl et al. 1993). In cases where measurements were doubtful and also in order to confirm the presence of faint lines, spectra obtained with the AURELIE spectrograph of the Haute Provence Observatory in July 1997, were examined. No variations were found, at least for the lines studied. The spectral resolution of these AURELIE spectra is 10000 and the signal to noise ratio similar to that of the spectrum used in the present work.

The main requirement of the SAC method to be applied is the presence of a large number of emission lines of different multiplets of the same ion which form in the same region of the stellar envelope. P Cygni shows an optical spectrum that is quite rich in emission lines (permitted and forbidden), including in particular many lines of N II and Fe III. The permitted transitions from less excited levels are accompanied by blueshifted absorption components, while the lines from more excited levels are, like the forbidden lines, purely in emission (Stahl et al. 1991; Stahl et al. 1993; Markova \& de Groot 1997). To increase the number of lines in our sample we decided to analyse in addition to the permitted lines with a pure emission profile also those with a P Cygni-type profile. Thus the sample of lines, we were able to analyse by the SAC method, consists of 30 lines from 7 multiplets of N II and 42 lines from 11 multiplets of Fe III. The multiplets as well as the distribution of the lines by multiplets are given in Cols. 1 and 6 of Table 1 .

To construct the SAC of a given multiplet one needs to know the lower-level statistical weight, $g$, the oscillator strength, $f$ and the dereddened emission flux, $F_{\lambda}$, of each line from this multiplet. In the present analysis we have used net emission equivalent widths (i.e. equivalent widths corrected for photospheric absorption), which we converted into line fluxes using a dereddened continuum calculated from $B=5.14$ and $V=4.72$ and deredenned with $E(B-V)_{\mathrm{PCyg}}=0.63$. In deriving the net emission equivalent widths $\left(W_{\lambda}\right)$ of the lines from their observed profiles, we were confronted by the following problems:

(i) possible contamination by photospheric absorption;

(ii) overlapping between wind absorption and emission in the case of lines with a P Cygni-type profile;

(iii) blending effects between pure emission lines which are not well-resolved in the spectrum.

The correction for photospheric absorption was performed by subtracting a synthethic spectrum, derived, using model calculations of Israelian (1995), from the observed spectrum. The spectrum, obtained in this way, was then measured for the equivalent widths of the lines. It must be noted here, that we were not fully convinced that such corrections had to be done, nor whether they were valid. This is because Markova et al. (2001) showed that the wind of P Cygni is probably sufficiently optically thick for the star to form a permanent pseudophotosphere. If this is the case, the observed profiles do not need to be corrected for photospheric absorption. However, Markova \& de Groot (1997) found that the emission peaks of the P Cygni profiles of Balmer, He I and N II lines in their 1990 spectra, were red-shifted with respect to the systemic velocity of the star, $V_{\text {sys }}=-22 \mathrm{~km} \mathrm{~s}^{-1}$, a result that can be easily explained if one believes that these peaks are distorted by photospheric absorption. In addition, Israelian (1995) argued that the complex structure of [Fe II] forbidden lines in P Cyg's spectrum is due to blending effects caused by weak photospheric lines. The results outlined above can be reconciled, if one suggests that the wind opacity can change significantly, a conjecture that seems quite reasonable. In fact Markova et al. (2001) showed that the mass loss rate of P Cygni can vary within $26 \%$ on a timescale of at least 600 days. In any case the photospheric correction has a small effect on our results

The contribution of wind absorption to the P Cygni-type profiles was accounted for by means of two methods, both of which turned out to give similar results as far as SAC slopes are concerned. The first way we proceeded was to measure the flux of the red part of each profile, integrating the emission flux redward of the emission peak, and multiplying the value obtained by 2 . This approach seems to be appropriate for our case since emission components are expected to be symmetric with respect to their peak intensity (Markova \& de Groot 1997). The second way we proceeded was to fit various Gaussians to the observed P Cygni profiles. It turned out that three Gaussians of different $F W H M$ (Full Width at Half Maximum) - two centered at $V_{\text {sys }}$ for the emission and one for the absorption - are usually enough, as a first approximation, to obtain a satisfactory fit. The contribution of absorption at velocities of more than $V_{\text {sys }}$ in this type of fit is small.

To separate and measure blends between pure emission lines we used Gaussian fits to the profiles. This fitting was performed taking into account the velocity and $F W H M$ of the lines. Blending was also considered taking into account the components's location on the self-absorption curve. Two examples of blend separations are shown in Fig. 1. The numbers given in the upper right hand corner of the figure denote the wavelength of the central position, the flux and the Gaussian width (in $\AA$ ) of each component. Let it be noted that not only the differences in line flux, represented by a dashed line, between the fit (dotted line) and the observations (solid line), are very small, but also that the central velocities and the FWHM of the three N II lines (multiplet 36), as determined by the fit, are in perfect agreement with each other as would be expected 

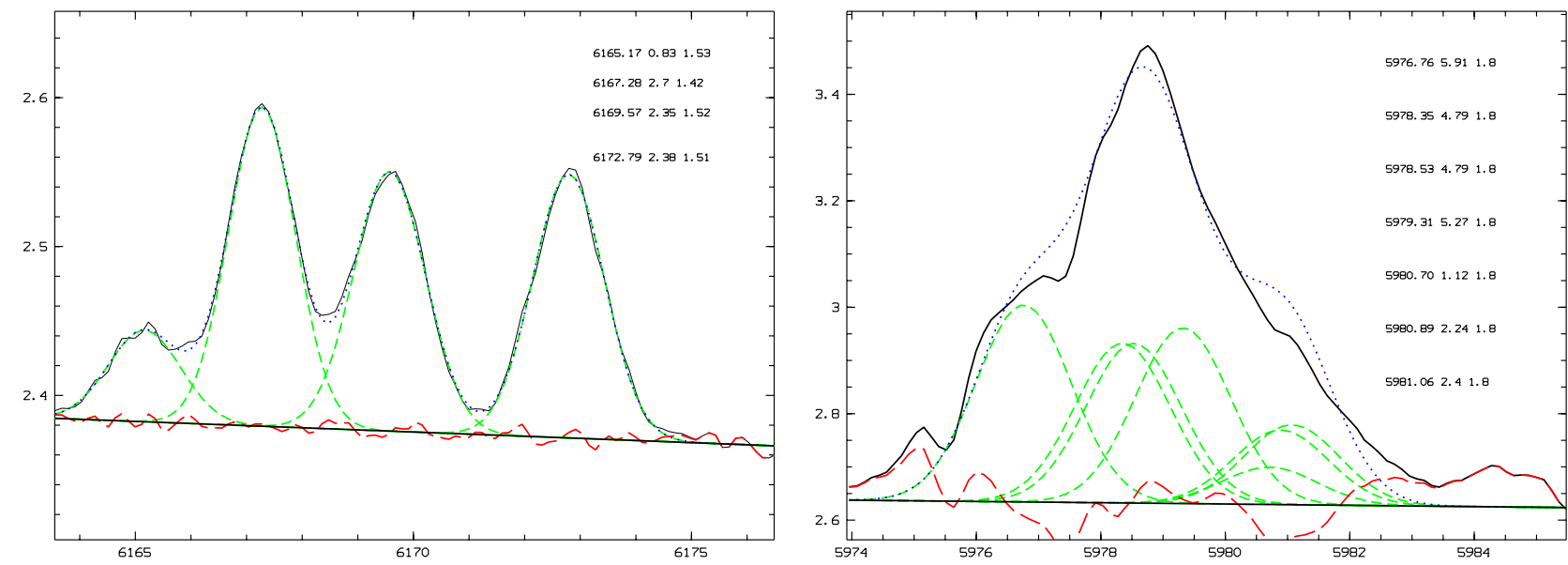

Fig. 1. Two examples of line fitting using Gaussians: left side, fitting of four weakly blended lines; right side, fitting of seven severely blended lines, six of which are from multiplet "756" whose SAC is displayed in Fig. 2.

if the lines were formed in the same region of the wind. The equivalent widths of the single pure emission lines were measured, fitting their profiles with a Gaussian.

\section{Application of the SAC method to P Cyg}

At the beginning of this section we would like to note that up to now the SAC method has, in all published papers except for conference proceedings, been applied to study the emission spectrum of the Fe II ion only. In the present study, we shall for the first time try to apply this method to the spectrum of Fe III and N II.

In our present work we have firstly tested that the points of the graphs of $\log \frac{F \lambda^{3}}{g f}$ against $\log (g f \lambda)$ for each multiplet lie near a curve. The slopes of the curves were then compared for different multiplets. The graphs with linear least square solutions for different multiplets are plotted in Figs. 2 and 3

Among these plots are two for different groups of very highly excited Fe III lines, those with lower to upper excitation potentials from 22.54 to $24.59 \mathrm{eV}$ being called "multiplet 705" and those with the corresponding excitation potentials from 23.61 to $25.68 \mathrm{eV}$ being called "multiplet 756 ". The results of the least squares solutions, where at least three lines are present, are given in Table 1.

The first thing we see is that the vertical dispersions of the points around the linear solutions are in most cases not very large in the same multiplet. The rms (root mean square) deviation is less than 0.4 for all multiplets except multiplets 756 of Fe III and 46 of N II. Secondly, the slopes of the graphs, found by linear least squares solutions, are still large for more excited multiplets, indicating that their lines are optically thick, even though their blue shifted absorption is not detectable. This is still true if only the more significant solutions are taken, for which the values of $\log (g f \lambda)$ of some lines in the same multiplet differ by more than 0.5 . There are even signs of a slope increase for the most excited multiplets. The range of $\log (g f \lambda)$ values hardly changes between the least and the most excited multiplets of N II, while in the case of Fe III, the $\log (g f \lambda)$ values are however considerably less for the first 3 multiplets. The slopes and other relevant information are listed in Table 1.
Table 1. Results from the SAC analysis.

\begin{tabular}{llllll}
\hline \hline $\begin{array}{l}\text { ion + } \\
\text { multiplet }\end{array}$ & $\begin{array}{l}\text { excit } \\
\text { lower }\end{array}$ & $\begin{array}{l}\text { potent } \\
\text { upper }\end{array}$ & $\begin{array}{l}\text { SAC } \\
\text { slope }\end{array}$ & rms & lines \\
\hline Fe III 4 & 8.2 & 11.1 & -0.40 & 0.2 & 4 \\
Fe III 5 & 8.6 & 11.1 & -0.16 & 0.2 & 8 \\
Fe III 68 & 14.1 & 16.5 & 0.00 & 0.2 & 3 \\
Fe III 113 & 18.2 & 20.6 & -0.35 & 0.2 & 7 \\
Fe III 114 & 18.4 & 20.6 & & & 2 \\
Fe III 115 & 18.7 & 20.9 & & & 2 \\
Fe III 117 & 18.8 & 20.9 & -0.42 & 0.3 & 3 \\
Fe III 118 & 20.6 & 23.6 & -0.44 & 0.2 & 4 \\
Fe III 119 & 20.6 & 23.7 & & & 2 \\
Fe III 705 & 22.5 & 24.6 & & & 2 \\
Fe III 756 & 23.6 & 25.7 & -0.80 & 0.4 & 5 \\
& & & & & \\
N II 3 & 18.5 & 20.7 & -0.44 & 0.1 & 6 \\
N II 5 & 18.5 & 21.2 & -0.62 & 0.2 & 6 \\
N II 19 & 20.7 & 23.1 & -0.70 & 0.2 & 3 \\
N II 20 & 20.7 & 23.2 & -0.21 & 0.1 & 4 \\
N II 28 & 21.2 & 23.2 & -0.81 & 0.15 & 4 \\
N II 36 & 23.1 & 25.1 & -0.81 & 0.1 & 3 \\
N II 46 & 23.2 & 25.2 & -0.86 & 0.40 & 4 \\
\hline
\end{tabular}

\section{Discussion}

The origin of the emission spectrum of P Cygni was partly discussed by Wolf \& Stahl (1985) who suggested that the upper terms of multiplets 115 and 117 of Fe III were pumped by two lines of He I. Pumping by two UV transitions in He I ( $\lambda \lambda$ 522, 537), followed by downward cascades, was suggested by Markova \& de Groot (1997) to explain the Fe III emission lines of multiplets $113,114,118$ and 119 . In addition, the authors noted that the $\mathrm{N}$ II multiplets with the highest upper term excitation potential $(\sim 25 \mathrm{eV})$ might be populated by dielectronic recombination, but the temperature of the wind seems to be too low for this mechanism to work. Stahl et al. (1993) however suppose that the highest excitation N II multiplets are excited by an unknown pumping mechanism.

The optical thickness of the more excited Fe III and N II lines without blueshifted absorption components, established 

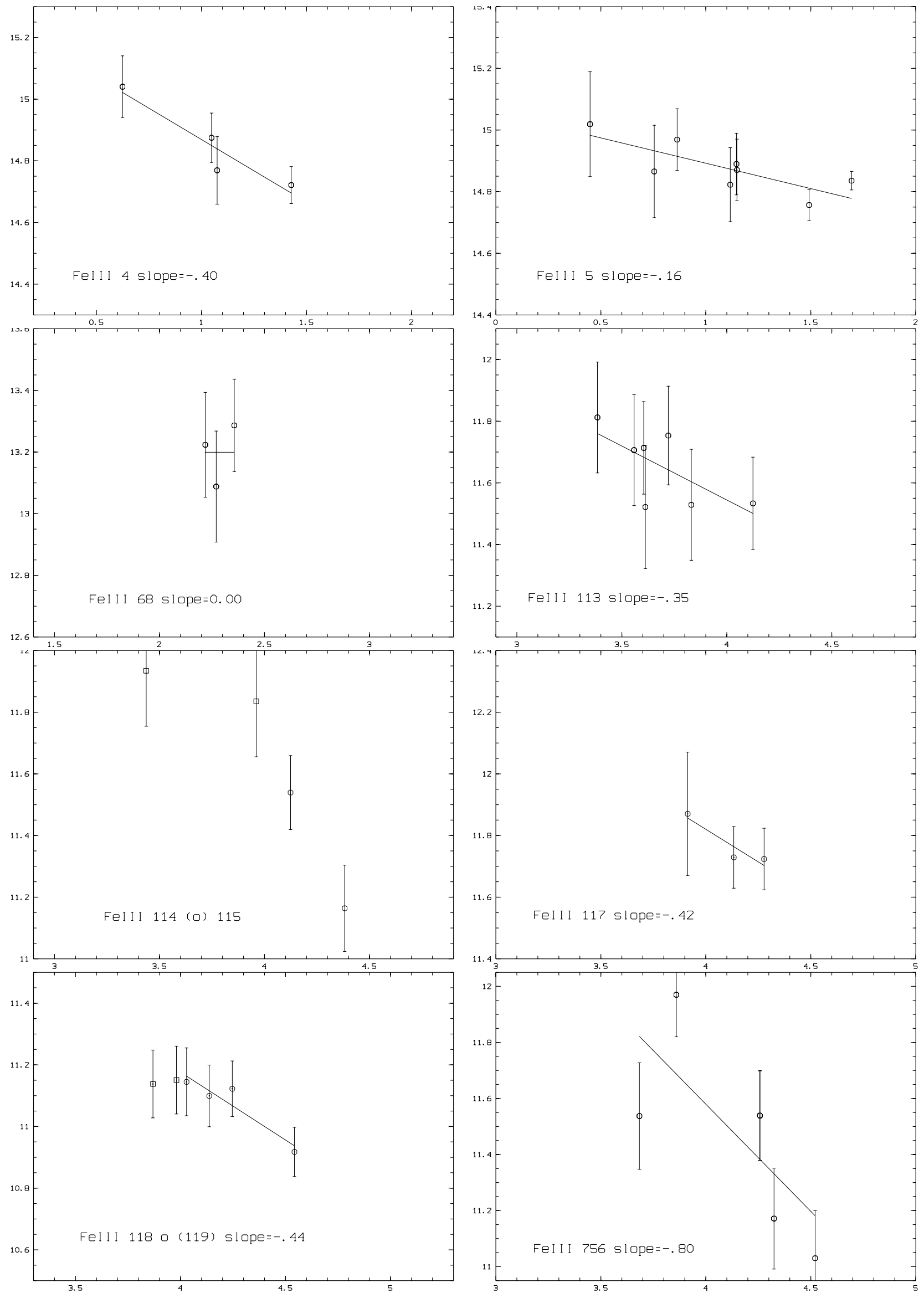

Fig. 2. Plots of $\log \frac{F \lambda^{3}}{g f}$ against $\log (g f \lambda)(\mathrm{SAC})$ for Fe III multiplets 4, 5, 68, 113, 114, 115, 117, 118, 119 and "756". 

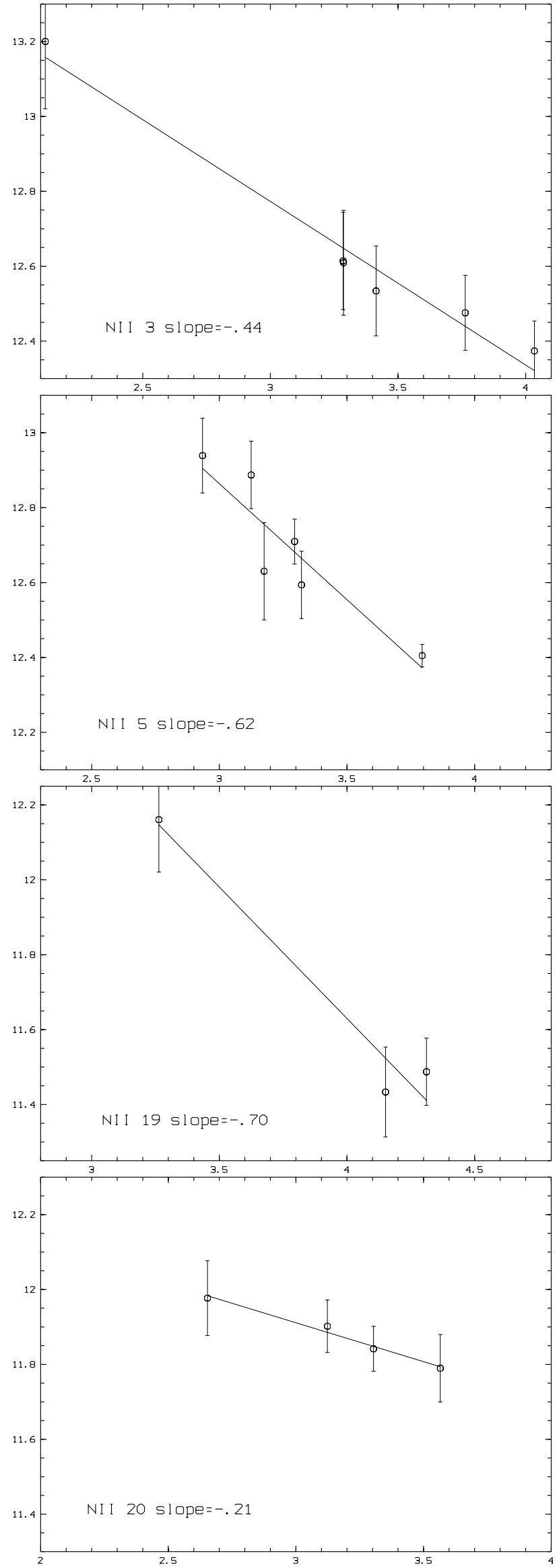

Fig. 2. continued

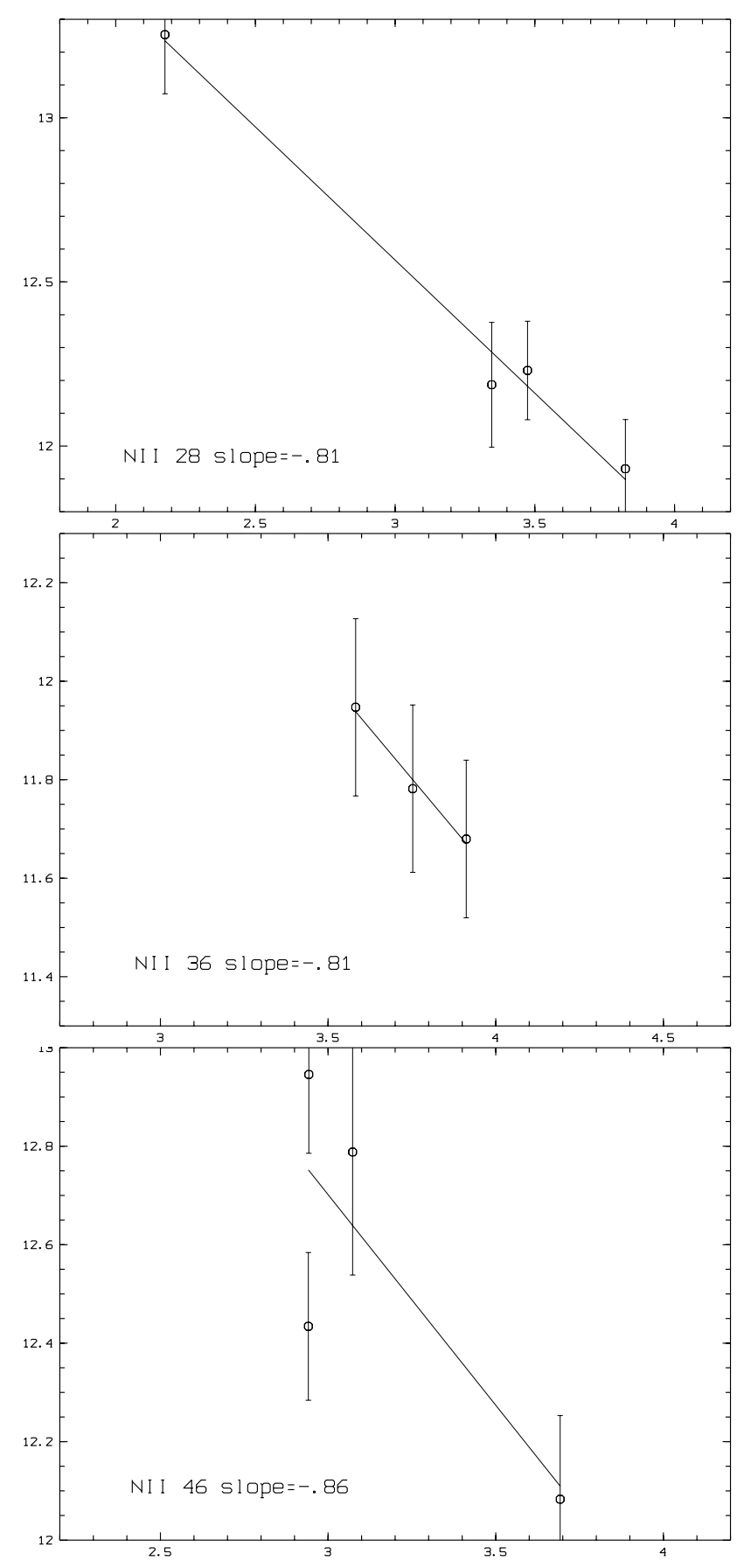

Fig. 3. Plots of $\log \frac{F \lambda^{3}}{g f}$ against $\log (g f \lambda)$ (SAC) for N II multiplets 3, $5,19,20,28,36,46$.

by us, is quite surprising, while the indications of an increase of SAC slope with increasing excitation, if confirmed in future work, would be in fact counter-intuitive. At the same $\log (g f \lambda)$ more excited lines are expected to be in any case optically thinner. A conspiracy of differential non-LTE effects appears unlikely as an explanation, especially when the points for a multiplet do not show large deviations from a curve. However we should note, that it is hard to rigorously test for such an effect, as both the upper and lower levels of the observed optical Fe III and N II lines could in principle show deviations from LTE. In objects showing many Fe II emission lines, the lower levels of 
strong lines are very often in LTE and in that case one only needs to test for effects depending on the upper level.

Another explanation, which could be suggested, will not work either, if the increase of curve slope at the same $\log (g f \lambda)$ for more excited multiplets is confirmed in future work. One might suppose that the more excited lines are only emitted from certain parts of a spherically symmetric wind. In that case the absence of blueshifted absorption components for excited multiplets might be explained by a large source function, so the emission in the lines per unit surface area, would be larger than that of the photosphere. In that case extra emission instead of absorption would be present on the blue side of the more excited lines, resulting in a blueshift of the mean radial velocity, as was in fact observed by Markova \& de Groot (1997). Such a blueshift could be particularly important if part of the receding material was behind and so occulted by the photosphere, but the symmetry of the lines, noted by Markova and de Groot, would suggest that such occultation is small. In any case if the wind has spherical symmetry, the less excited lines could not be optically thinner, because those lines would also be emitted in the region of emission of the more excited lines, where they should not be optically thinner than those excited lines.

If we exclude the previous explanations, we may need to conclude that the wind of P Cyg is anisotropic. Optically thick excited lines with no blue shifted absorption components would in that case be mainly formed in clouds, which either have a large source function or which do not occult much of the photosphere. The optically thinner lower excitation lines with blueshifted absorption, could then be mainly formed in large regions of the wind, covering the photosphere, with only a small optically thicker contribution to their emission from the clouds. The physical reason for such a situation is however not immediately clear. Heating due to shock waves might occur in the clouds, producing extra ionizing radiation, followed by more recombinations and cascades plus pumping to extremely excited levels Such a situation might also be a way of explaining the presence of the most excited N II lines. Theoretical work is required to see whether this is a viable option.

We must also finally point out that effects such as an increase in self-absorption curve slope for more excited multiplets, would be hard to find in detailed spectral syntheses, based on spherically symmetric wind models. Semi-empirical methods can be quite powerful in searches for unknown physical processes, present in relatively complex situations.

Acknowledgements. N. Markova is grateful for the warm hospitality of the Observatoire de Marseille (France). G. Muratorio and M. Friedjung wish to acknowledge the hospitality of the National Astronomical Observatory (NAO, Bulgaria), where part of the work was done. This study was supported by the French CNRS and the Bulgarian Academy of Sciences. Research at the NAO was in part supported by the NSF through grant F-813/1998 to the Bulgarian Ministry of Education.

\section{References}

Baratta, G. B., Friedjung, M., Muratorio, G., Rossi, C., \& Viotti, R. 1998, The Self Absorption Curve Method, A Users' Manual, IAS Internal Report, December 1998

Friedjung, M., \& Muratorio, G. 1987, A\&A, 188, 100

van Genderen, A. M. 2001, A\&A, 366, 508

de Groot, M., Sterken, C., \& van Genderen, A. 2001, A\&A, 376, 224

Humphreys, R. M. 1999, in Variable and Non-spherical Stellar Winds in luminous Hot Stars, ed. B. Wolf, O. Stahl, \& A. W. Fullerton (Springer), 243

Israelian, G. 1995, A\&A, 300, 834

Israelian, G., \& de Groot, M. 1999, Space Sci. Rev., 90, 493

Lamers, H. J. G. L. M. 1986, in Luminous Stars and Associations in Galaxies, ed. C. de Loore, \& A. Willis (Reidel, Dordrecht), IAU Symp., 116, 157

Markova, N., \& de Groot, M. 1997, A\&A, 326, 1111

Markova, N., Morrison, N., Kolka, I., et al. 2001, A\&A, 376, 898

Markova, N., Scuderi, S., de Groot, M., et al. 2001, A\&A, 366, 935

Muratorio, G., \& Friedjung, M. 1988, A\&A 190, 103

Muratorio, G., Viotti, R., Friedjung, M., Baratta, G. B., \& Rissi, C. 1992, A\&A, 258, 423

Stahl, O., Mandel, H., Szeifert, Th., Wolf, B., \& Zhao, F. 1991, A\&A, 244,467

Stahl, O., Mandel, H., Wolf, B., et al. 1993 , A\&AS, 99, 167

Stahl, O., Kaufer, A., Wolf, B., et al. 1995, J. Astron. Data, 1, dir. 3

Wolf, B., \& Stahl, O. 1985 , A\&A, 148, 412 